\title{
Muestreo comprimido en red inalámbrica de sensores para monitorización de vibración en puentes
}

\author{
Compressed Sensing in Vibration Monitoring \\ Wireless Sensor Network
}

Osvaldo Casares-Quirós'

Fecha de recepción: 2 de mayo del 2014 Fecha de aprobación: 7 de setiembre del 2014

Casares-Quirós, O. Muestreo comprimido en red inalámbrica de sensores para monitorización de vibración en puentes. Tecnología en Marcha. Edición Especial Movilidad Estudiantil 2014. Pág 


\section{Palabras clave}

Codificador Condicional; Decodificador Predictivo; Monitorización de Vibración en Puentes; Muestreo Comprimido; Redes Inalámbricas de Sensores, Submuestreo; Waspmote.

\section{Resumen}

Un 70\% de los puentes de Costa Rica presentan elementos críticos en la estructura. Las redes inalámbricas de sensores han sido utilizadas para la monitorización de estructuras debido al bajo tiempo de instalación y costo económico ya que el sistema no requiere alambrado.

En este documento se presenta una red inalámbrica de sensores capaz de ejecutar algoritmos de muestreo comprimido para medir la vibración en puentes. El diseño de la red se plantea buscando integridad en los datos y un reducido consumo energético.

El algoritmo realiza submuestreo mediante pares codificador-decodificador en el protocolo de comunicación. El par seleccionado es codificador condicional y decodificador predictivo. Esta combinación presenta elementos en común que se pueden compartir para obtener mejores estimaciones en pocos pasos.

Se presentan dos variantes del par codificadordecodificador: variable-fijo y fijo-variable. El primer caso propone una compresión de factor constante durante cada período de muestreo mientras que el segundo una compresión variable en función del comportamiento de la señal en el tiempo.

Tras una prueba experimental con Waspmotes se obtuvo que la variante fijo-variable presenta una reducción del $56.58 \%$ de consumo de potencia introduciendo un error máximo de $\pm 0.00195 \mathrm{~g}$ y una compresión del $52.44 \%$ de muestras. Con ello la red aumentó su autonomía energética de 17 horas a 26 horas y media. Mediante un análisis matemático la técnica variable-fijo presenta una reducción a lo sumo de $74.81 \%$ de consumo de potencia en transmisiones de los nodos sensores y compresión de un 90\% de muestras como máximo.

\section{Keywords}

Bridge Vibration Monitoring; Compressed Sensing; Conditional Coder; Downsampling; Predictive Decoder; Waspmote; Wireless Sensor Networks.

\section{Abstract}

Over $70 \%$ of the bridges in Costa Rica have critical elements in the structure. Wireless sensor networks have been used for structural monitoring because of their short installation time and low economic cost due to the lack of wiring.

In this research is presented a wireless sensor network which uses a compressed sensing algorithm for vibration monitoring on bridges. The network design is proposed expecting data integrity and energy harvesting.

The algorithm performs downsampling by coderdecoder pairs. The selected pair is conditional encoder and predictive decoder because this combination has elements in common that can shared to get better estimates in few steps.

Two coder-decoder pairs variants are presented: variable-fixed and fixed-variable. The first one proposes a constant factor compression during each sampling period while the second presents variable compression that depends of the signal behavior over time.

After an experimental test using Waspmotes the fixed-variable variant has a $56.58 \%$ reduction of power consumption by introducing a maximum error $\pm 0.00195 \mathrm{~g}$ and compress in $52.44 \%$ the amount of samples. This algorithm increased the network energy autonomy from 17 hours to 26.5 hours. Through mathematical analysis, the variablefixed technique reduces in $74.81 \%$ the power consumption in sensing nodes transmissions and decrease in $90 \%$ the number of samples. 


\section{Introducción}

Cerca del $70 \%$ de los puentes en Costa Rica presentan elementos críticos en la estructura (MOPT, 2002). Desde el año 2007 se inició la evaluación del estado de los puentes para promover el desarrollo de capacidad en la planificación de rehabilitación, mantenimiento y administración de puentes (Agencia de Cooperación Internacional de Japón, 2007). En los últimos 5 años el tema de puentes ha sido un problema grave de interés nacional, incluyendo pérdidas humanas por fallas. Además, durante los años 2012 y 2013 puentes en las principales rutas nacionales han colapsado e impactado de manera negativa el desarrollo del país.

En el año 201 I el Instituto Tecnológico de Costa Rica incursiona con el proyecto de investigación eBridge "Predicción remota de fallas en puentes» que propone un sistema de monitorización de estructuras mezclando inspecciones visuales con redes sensoriales para complementar y revolucionar los análisis estructurales en el país. Una variable considerada por este grupo es la vibración (Ortiz, 2013).

Durante una prueba de carga realizada en 2012, el grupo recolectó datos de vibración durante 24 horas. No obstante, se encontró una limitante en la autonomía energética del sistema ya que se restringía a aproximadamente 17 horas. Por este motivo, las baterías de los nodos sensores tuvieron que ser cargadas durante la prueba para evitar la pérdida de datos (Libelium, 2013; Ortiz, 2013). Ante esta problemática, una reducción del consumo de potencia es necesario para aumentar el tiempo de duración de batería para aprovechar las características inalámbricas del sistema.

Diversos métodos de muestreo comprimido en redes inalámbricas de sensores han sido desarrollados en los últimos 6 años. Estas se basan en algoritmos de muestreo comprimido que son típicamente usados en el área de procesamiento digital de señales con el objetivo de reducir la cantidad de transmisiones en la red inalámbrica, lo que conlleva a una reducción significativa del consumo energético ya que la comunicación en radio frecuencia es crítica en estos sistemas (Barceló, 20 I2;Libelium, 20 I3).

\section{Monitorización de vibración mecánica}

La vibración mecánica en puentes es una variable que ha tomado mucha importancia en el área de monitorización de estructuras. Su principal objetivo es caracterizar mediante un análisis modal las formas de vibración. Por ejemplo, en los puentes se analizan los procesos relacionados a la estructura con el fin de obtener una descripción matemática del comportamiento dinámico. Tras el análisis se puede obtener la relación:

$$
M \ddot{x}(t)+C \dot{x}(t)+K x(t)=F(t)
$$

donde $M$ es la matriz $N \times M$ de masa, $C$ es la matriz $N x N$ de amortiguamiento y es la matriz de rigidez del material. $F$ es el vector $N x /$ de fuerza mientras que es el vector de desplazamiento. Los puntos sobre $x$ denotan el número de derivaciones con respecto al tiempo. Suponiendo que el vector $F$ está compuesto de señales senoidales como una serie de Fourier, por linealidad, se sabe que $x$ también lo es. Al transformar al dominio de frecuencia se obtiene de forma singular (Hardyniek, 2009):

$$
\left[-\omega^{2} M+j \omega C+K\right]\{x\} e^{j \omega t}=\{f\} e^{j \omega t}
$$

Para el análisis, las mediciones realizadas en función del tiempo por los sensores son transformadas al dominio de la frecuencia con lo que es posible la obtención de un modelo matemático del comportamiento dinámico del puente utilizando la ecuación - Ello se obtiene mediante una transformación discreta de Fourier donde típicamente se emplea el algoritmo de la transformada rápida de Fourier.

En este caso, la vibración es una señal dinámica que se presenta en puentes a bajos niveles de frecuencia para la monitorización de vibración ambiental, normalmente las componentes superiores a $25 \mathrm{~Hz}$ suelen ser filtradas. No obstante, las primeras armónicas se encuentran entre los $\mathrm{OHz}$ y los $15 \mathrm{~Hz}$ (Hardyniek, 2009; Wenzel y Pichler, 2005). Por ello, con base en el teorema de Nyquist una frecuencia de $30 \mathrm{~Hz}$ es suficiente para poder muestrear la señal.

\section{Red inalámbrica de sensores}

una red inalámbrica de sensores es un conjunto de nodos distribuidos en un espacio para medir y monitorizar variables. Dentro de la red, existen dos tipos de nodos: nodo sensor y nodo sumidero. El rol 
de nodo sensor se basa en realizar la medición de la variable de interés y llevar a cabo la transmisión de la información mediante algún protocolo de comunicación. El nodo sumidero se encarga de reunir las mediciones de nodos sensores (Tapia, 2006).

En cuanto al nodo sensor, este debe tener cuatro módulos de hardware principales: energía, lógica computacional y de almacenamiento, sensor y comunicación (Garbarino, 2006). El apartado energético es un parámetro muy importante ya que define la autonomía de la red y en muchos casos el tamaño del nodo. Una técnica para obtener ahorro de energía es mediante algoritmos en el protocolo de comunicación (Hu y Cao, 20l0).

Entre los estándares empleados en comunicaciones para redes inalámbricas de sensores sobresale el IEEE 802. I 5.4. Dada la latencia de I 5 ms y la frecuencia de muestreo de $30 \mathrm{~Hz}$ este es un factor de interés a la hora de implementar el nodo sumidero. Así mismo, el alcance de $10-20 \mathrm{~m}$ puede parecer reducido, sin embargo, en Costa Rica un 76\% de los puentes no supera los $30 \mathrm{~m}$ por lo que una topología en estrella satisface las condiciones de recolección de datos de una manera mínima de transmisiones dentro de la red. Para el 24\% restante, se puede implementar en topología árbol o distribuida para poder alcanzar la completa medición en el puente (Archundia, 2003; MOPT, 2002).

\section{Muestreo comprimido}

el muestreo comprimido es una técnica conocida en el procesamiento digital de señales desde 2006 (Donoho, 2006). Esta establece que una señal poco densa en algún dominio $\phi$ de $m$ dimensiones, donde $m$ es un número natural puede ser representada mediante una base ortonormal $\psi$ de tal forma que la cantidad de componentes sea óptima. Ello facilita a la compresión de la señal $x$ ya que requiere menos dimensiones sin introducir alto error a la señal (Donoho, 2006). En un sentido matemático se tiene:

$$
X \approx \sum_{i=1}^{k} s_{i} \psi_{i}=s \psi
$$

donde $k$ es un número natural tal que $k<<m$.

Las ventajas de aplicar el algoritmo de muestreo comprimido durante el procesamiento digital de señales radica en la reducción de información necesaria para reconstruir la señal. Esto se traduce en una reducción focalizada en cinco aspectos: frecuencia de muestreo, cantidad de datos, recursos para su almacenamiento, exigencia de velocidad en componentes físicos empleados y uso de ancho de banda para la transmisión de los datos (Fornasier y Rauhut, 2010).

\section{Algoritmos con muestreo comprimido}

cabe resaltar que una red inalámbrica de sensores siempre se puede modelar como un arreglo matricial de poca densidad por lo que la aplicación de la técnica se ve favorecida (Xiaoyan, Houjun yZhijian, 20 I0). Los fundamentos del muestreo comprimido se aplica a las redes inalámbricas de sensores para mejorar la técnica de adquisición de datos en redes amplias (Haifeng, Zhen y Jianmin, 20 I2; Zhang, chen y Liu, 2009). Con base en las prácticas de diseño la red debe ser escalable y el tráfico de datos puede aumentar conforme se evoluciona la red (Xue, Donf y Shi, 2013). Con el muestreo comprimido si el número de nodos transmitiendo aumenta, el consumo de potencia de la red aumenta de manera sublineal por lo que ayuda a ahorrar energía especialmente en redes con alta cantidad de nodos (Bajwa, Haupt, Sayeed y Nowak, 2006). Es importante conocer que no todos los nodos pertenecientes a la red deben realizar el algoritmo ya que en algunos casos es contraproducente (Luo, Xiang y Rosenberg, 2010).

Tras la iniciativa de emplear muestreo comprimido en redes inalámbricas de sensores se ha combinado con otras técnicas en el protocolo de comunicación para aumentar su efectividad (Li, Zhang, Wu y Zhou, 2013; Nguyen, Jones y Krishnamurthy, 20I0; You, Han y Su, 20 I3). La clave radica en buscar reducir la cantidad de transmisiones dentro del sistema ya que representan el mayor porcentaje de consumo energético (Balouchestani, 201 I; Balouchestani, Raahemifar y Krishnan, 201 I). Algunos modelos probabilísticos son utilizados de manera complementaria al algoritmo con el fin de obtener mejores resultados en la reconstrucción de la señal (Liu, Liu y Qiu, 20 I 0; Hu y Yang, 20 I0; Meng, Li y Han, 2009). La selección de cuáles técnicas emplear tanto para la adquisición de datos como para la reconstrucción de la señal dependen completamente de la naturaleza de la misma. Ello afecta directamente al desempeño del algoritmo en términos de consumo 
de potencia y calidad de la señal reconstruida (Yang Xiao y Zhang, 2013). Así mismo, la topología de red empleada define la forma óptima para la captación de la señal (Haupt, Bajwa, Rabbat y Nowak, 2007).

El fundamento matemático del muestreo comprimido se aplica suponiendo que la señal es la matriz de dimensióncuyos coeficientes son los valores medidos por cada nodo sensor. Suponiendo que la red es de sensores, la señal tiene dimensiones, cada una representada por un sensor. Mediante el algoritmo, se define la cantidad de dimensiones que se van a medir, donde, por lo que es recomendable para arreglos de alta cantidad de sensores. Las mediciones realizadas se envían a través de comunicación inalámbrica a un nodo sumidero y este se encarga de reconstruir las faltantes dimensiones. Ello ayuda a reducir el consumo de potencia de transmisiones, y como, las transmisiones se reducen cerca del $90 \%$. No obstante, la lógica en los nodos sensores es más compleja lo que aumenta el consumo de potencia de procesamiento. Pese a ello, la relación de consumo de potencia por procesamiento es casi despreciable en comparación con el consumo por la comunicación inalámbrica (Barceló, 20|2).

\section{Submuestreo mediante pares codificador-decodificador}

La técnica clásica de submuestreo aplicada a protocolos de comunicación se basa en un par codificador-decodificador que reduce la cantidad de información a transmitir. Las etapas de este método se muestran en la figura I. El proceso describe cómo, de la señal medida se obtiene la señal reconstruida (Barceló, 2012).

Un codificador conocido es el de submuestreo condicional. Este emplea información pasada para identificar patrones en la señal. Se basa en predicciones comparadas con la medición real para determinar si se efectúa la adquisición de la muestra.
El decodificador predictivo es aquel que requiere de información anterior, además de la muestra actual. Los valores no recibidos se completan mediante un valor predicho mediante algún cálculo matemático que se ajuste a la naturaleza de la señal.Típicamente la predicción se realiza mediante estadística basada en promedio y moda, no obstante, también puede ser mediante procesos estocásticos basados en correlación.

El par codificador condicional-decodificador predictivo comparte el uso de predicciones. Para ello, el nodo sensor realiza una etapa de entrenamiento donde se identifica el comportamiento básico en el entorno para realizar estimaciones más acertadas. Se define $\Delta$ como el error máximo permitido para una aproximación. Mediante este tipo de submuestreo se proponen los dos algoritmos: $\Delta$ fijo-Kvariable y $\Delta$ variable-Kfijo.

El método $\Delta$ variable-Kfijo se basa en la técnica pura del muestreo comprimido a redes inalámbricas de sensores. Presenta la ventaja que la cantidad de datos recopilados es constante a lo largo del tiempo, es decir, la energía consumida por transmisiones en la red se reduce en un factor de N-K. No obstante, presenta el inconveniente de que necesita una retroalimentación del nodo central por lo que en cada proceso de medición el nodo sensor debe recibir información a través de la red inalámbrica.

La técnica $\Delta$ fijo-Kvariable suprime el lazo de retroalimentación para que la red inalámbrica sea unidireccional. La limitación que presenta es la incapacidad de determinar cuántos nodos transmitirán la medición en cada ejecución.

\section{Prueba de algoritmos de compresión}

Durante una prueba de estudio del grupo de investigación eBridge, se pudo identificar la cantidad de tráfico que viaja a través del puente sobre el río Purires en la ruta 2. En dicha prueba se observó que en promedio transita I eje cada 7.5s, es decir, la fre-

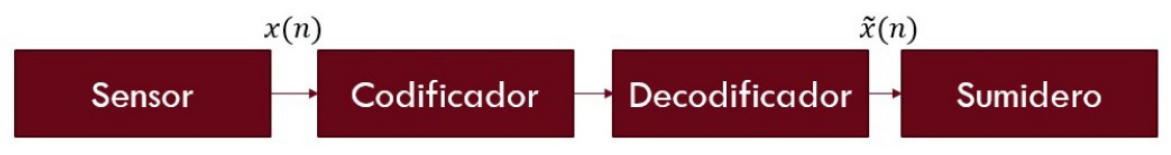

Figura I. Diagrama de bloques de un protocolo de comunicación basado en codificador-decodificador. 
cuencia de actividad promedio es 0,13Hz. Además, con la velocidad y longitud promedio de cada vehículo se aproximó que cada uno excita el puente por aproximadamente 3s. (Ortiz, 20 I3).

Para evaluar el desempeño del algoritmo $\Delta$ fijo-Kvariable la prueba consiste en el nodo sensor sujeto a una superficie donde se aplicaron pulsos con una frecuencia de $0.13 \mathrm{~Hz}$ y una duración de 3 s simulando la actividad en el puente de estudio. Durante el proceso, se medió la aceleración en los 3 ejes de coordenadas cúbicas y el consumo de potencia del nodo sensor, esto con el fin de analizar las dos variables de interés: integridad de datos y consumo de potencia. En cuanto al algoritmo $\Delta$ variable-Kfijo se hará un análisis teórico para cumplir la condición de $K$ «N .

La medición de aceleración se realizó mediante los nodos Waspmote y el consumo de potencia mediante una unidad de medida de fuente (SMU).

\section{Resultados y análisis}

En la figura 2 se muestra el consumo de corriente del nodo sensor sin algoritmo de compresión mientras que la figura 3 presenta el caso con el método $\Delta$ fijo-Kvariable.

En la figura 3 se identifica dos partes importantes para el análisis: entrenamiento (I) y ciclo (2). En el cuadro I se compara el consumo de corriente durante ambas etapas para el sistema en los dos casos medidos.
El entrenamiento es el aprendizaje para reconocimiento del patrón de medición en la red. Dado el agregado de los cálculos para la etapa de predicción, el consumo es ligeramente mayor cuando se emplea el algoritmo de compresión. No obstante, esta etapa se ejecuta únicamente los primeros $5 \mathrm{~s}$ por lo que en un análisis global de desempeño es casi despreciable. En contra parte, el ciclo se ejecuta después del entrenamiento hasta que se termine la medición. Note que el consumo es reducido en un $56,58 \%$ durante esta etapa, la cual al ejecutarse durante todo del tiempo después de los $5 \mathrm{~s}$ de estudio, se puede aproximar como el ahorro energético total de la red.

Un modelo $\Delta$ fijo-Kvariable presenta algunos casos donde se estaría muestreando sin recibir inclusive una medición en el nodo sumidero, sin embargo, durante una excitación al puente todos los nodos estarán transmitiendo. En contraparte, suponiendo que $N \approx$ I $O K$ el consumo con la técnic $\Delta$ variable-Kfijo se puede aproximar como:

Lo que reduce el consumo de potencia en un $74,81 \%$ por nodo. Esta reducción se cumple bajo los términos estrictos desde el punto de vista de transmisión de datos. Cabe recordar que la técnica $\Delta$ variable-Kfijo requiere de gasto de energía que $\Delta$ fijo-Kvariable no sufre en el momento de recepción de datos. En el método variable-fijo no se puede realizar una desconexión del transceptor mientras no se transmite ya que también puede recibir información. La técnica $\Delta$ variable-Kfijo es

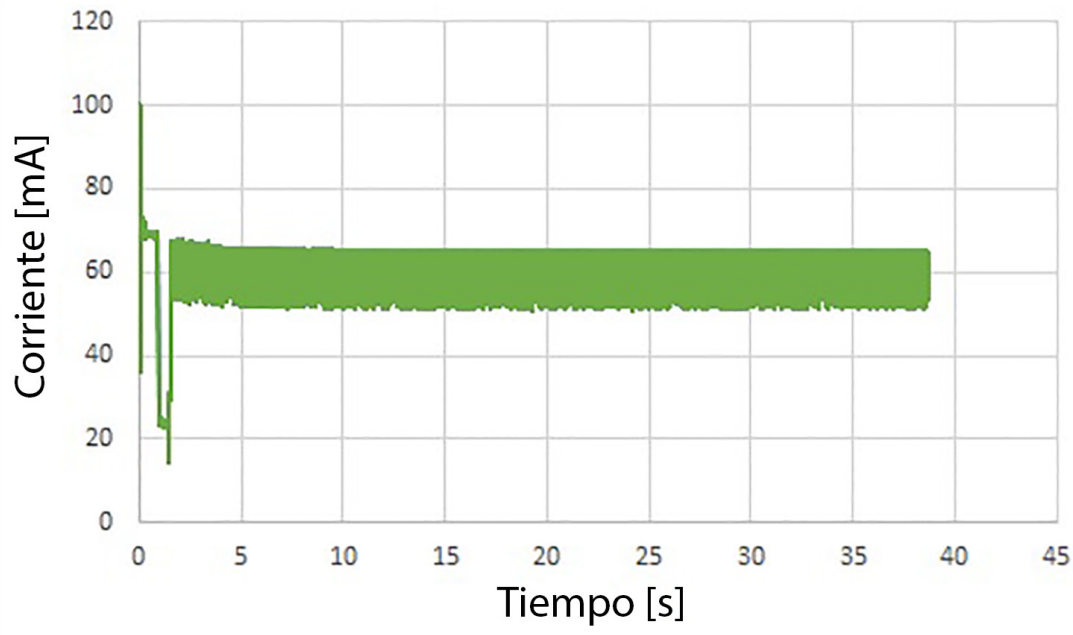

Figura 2. Consumo de corriente del nodo sensor con fuente fija a $3.795 \mathrm{~V}$ sin algoritmo de compresión. 


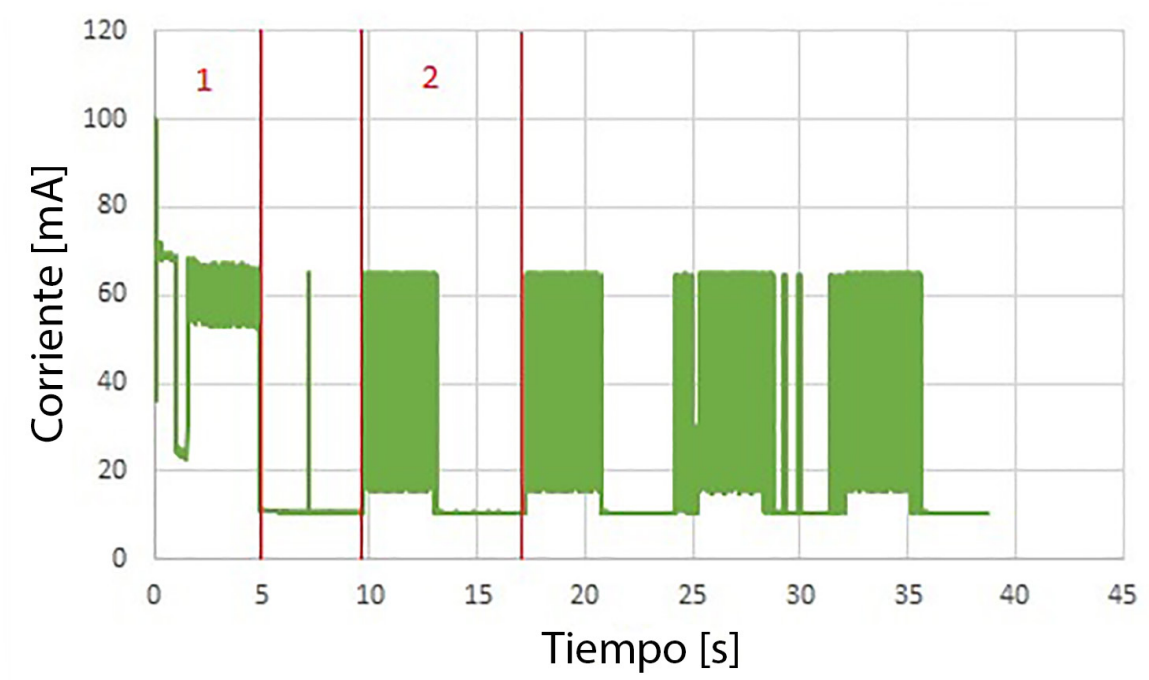

Figura 3. Consumo de corriente del nodo sensor con fuente fija a $3.795 \mathrm{~V}$ con el método fijo-variable

Cuadro I. Comparación de consumo de corriente.

\begin{tabular}{|c|c|c|c|c|c|}
\hline \multicolumn{3}{|c|}{ Entrenamiento $(\mathrm{I})$} & \multicolumn{3}{c|}{ Ciclo (2) } \\
\hline $\begin{array}{c}\text { Sin compresión } \\
{[\mathrm{mA}]}\end{array}$ & $\begin{array}{c}\Delta \text { fijo } \\
\text { Kvariable }[\mathrm{mA}]\end{array}$ & $\begin{array}{c}\text { Porcentaje } \\
{[\%]}\end{array}$ & $\begin{array}{c}\text { Sin Compresión } \\
{[\mathrm{mA}]}\end{array}$ & $\begin{array}{c}\Delta \text { fijo } \\
\text { Kvariable } \\
{[\mathrm{mA}]}\end{array}$ & $\begin{array}{c}\text { Porcentaje } \\
{[\%]}\end{array}$ \\
\hline 60,53 & 61,39 & 1,41 & 63,11 & 27,40 & $-56,58$ \\
\hline
\end{tabular}

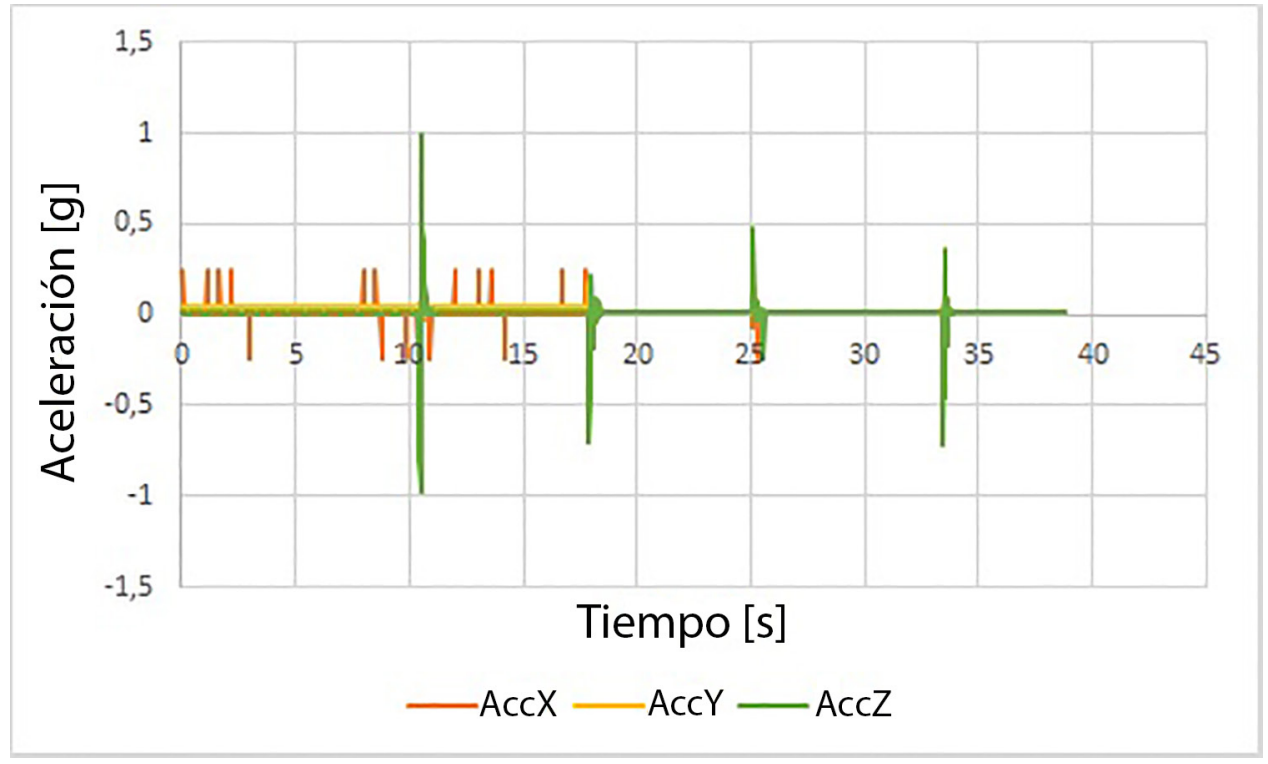

Figura 4. Medición de aceleración sin algoritmo de compresión. 
recomendable si se utilizan transceptores donde el consumo de corriente por transmisión es mucho mayor que al de recepción.

Las muestras recopiladas por el nodo sensor sin el algoritmo de muestreo comprimido se presentan en la figura 4, mientras que la señal reconstruida mediante $\Delta$ fijo-Kvariable se muestra en la figura 5 .

Note que ambas señales son similares, presentan perturbaciones significativas cada 7,5s aproximadamente. No obstante, en la figura 4 se puede observar entre los 0 y I7s que en el eje $X$ también se midió pequeñas perturbaciones con la misma magnitud. Es evidente que estos valores son indicios de ruido en la medición ya que son de misma magnitud y aparecen cuando no se aplicó ningún estímulo al sensor. Este comportamiento es atribuido al gran flujo de datos en la red, el buffer de datos se satura y provoca lecturas incorrectas como en este caso. Esto se puede corregir empleando filtros o algoritmos de corrección de datos. En la figura 5 se observa que este error no existe, sino que se limita a registrar los eventos sucedidos a $0,13 \mathrm{~Hz}$ ya que el par codificador-decodificador funciona a su vez como un filtro digital.

Con respecto a la cantidad de datos, en promedio al muestrear en un ciclo se manejaron 225 datos, al aplicar el algoritmo $\Delta$ fijo-Kvariable se reduce a I 8 y con la técnicavariable-fijo a 22,5. La reducción de datos alcanza el $52,44 \%$ y el $90 \%$ respectivamente para cada método.

En cuanto a la integridad de los datos, en las partes dinámicas el error introducido por el algoritmo $\Delta$ fijo-Kvariable es cero ya que ante perturbaciones se muestrea a la tasa máxima definida por la transmisión. No obstante, en período de reposo, el algoritmo introduce un margen de error de $\pm 0,00195 \mathrm{~g}$, lo cual es inclusive menor que el inducido por el sensor $( \pm 0,002 g)$. En el caso con $\Delta$ variable-fijo el error es variable pero presente y diferente en $\mathrm{N}-\mathrm{K}$ muestras en cada período.

\section{Conclusiones}

Los pares codificador condicional-decodificador predictivo en redes inalámbricas de sensores son usados como herramienta de submuestreo para disminuir el costo energético en redes inalámbricas de sensores en términos de transmisión de datos. Además, el uso de predicciones favorece que el error inducido por el algoritmo sea reducido.

La naturaleza de la señal a medir determina la efectividad de diferentes variaciones de la técnica. Para el caso de monitorización de vibración en puentes, la señal dinámica requiere una tasa de muestreo como mínimo de $30 \mathrm{~Hz}$. Además, el comportamiento en función del tiempo se debe analizar para conocer el

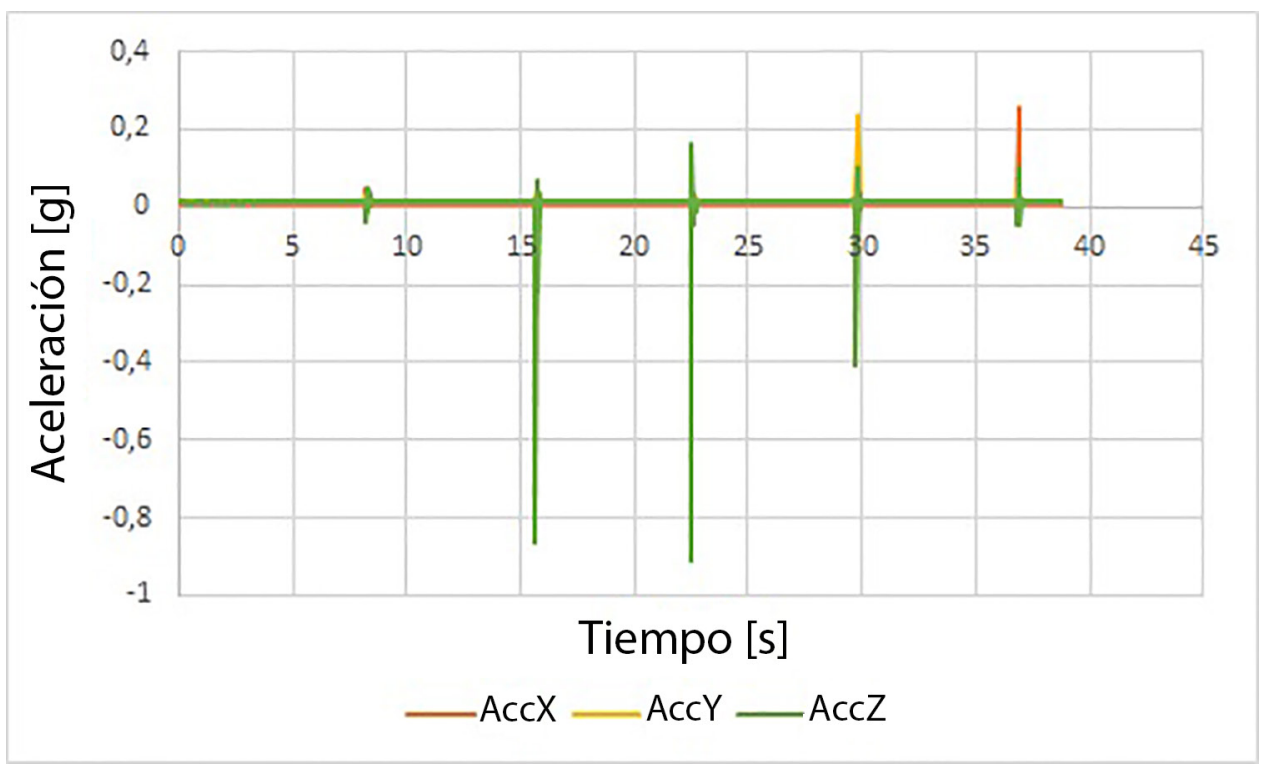

Figura 5. Medición de aceleración con método defijo-variable. 
momento conveniente para llevar a cabo la etapa de estudio.

La variante $\Delta$ fijo-Kvariable presenta una reducción del $56,58 \%$ de consumo de potencia. La técnica $\Delta$ variable-Kfijo presenta una reducción a lo sumo de $74,81 \%$ de consumo de potencia en transmisiones de los nodos sensores, no obstante, es significativo el consumo de potencia por recepción de datos.

En el caso $\Delta$ fijo-Kvariable la cantidad de datos se reduce en un $52,4 \%$ induciendo un error máximo de $\pm 0,00195 \mathrm{~g}$ mientras que $\Delta$ variable-Kfijo comprime hasta un $90 \%$ con un error variable.

\section{Referencias}

Agencia de cooperación internacional de Japón. (Febrero 2007). El estudio sobre el desarrollo de capacidad en la planificación de rehabilitación, mantenimiento y administración de puentes basado en 29 puentes de la red de carreteras nacionales en Costa Rica. Reporte técnico, MOPT.

Archundia, F. (2003). Wireless personal area network (wpan) and home networking. Tesis de maestría, Universidad de las Américas Puebla.

Bajwa, Haupt, Sayeed y Nowak. (2006). Compressive wireless sensing. IPSN'06.

Balouchestani, B. (20II) Low-power wireless sensor network with compressed sensing theory. IEEE.

Balouchestani, Raahemifar, y Krishnan. (20II). Increasing the reliability of wireless sensor network with a new testing approach based on compressed sensing theory. IEEE.

Barceló, J. (20 I2). Communications in Wireless Sensor Networks: Compression, Energy Efficiency and Secrecy. Tesis de doctorado, Universitad Autónoma de Barcelona.

Donoho, D. (2006). Compressed sensing. IEEE Transactions on Information Theory, 52(4).

Fornasier y Rauhut. (20।0). Compressive sensing.

Garbarino, J. (2006). Protocolos para redes inalámbricas de sensores. Tesis de maestría, Universidad de Buenos Aires, Noviembre 201।.

Haifeng, Zhen y Jianmin. (2012). Wavelet transform-based distributed compressed sensing in wireless sensor network.

Hardyniek, A. (2009). Dynamic testing and modeling of a superelevated skewed highway bridge. Tesis de maestría, Virginia Polytechnic Institute and State University.
Haupt, Bajwa, Rabbat y Nowak. (2007) Compressed sensing for networked data.

Hu y Cao. (2010). Wireless Sensor Networks. CRC Press.

Hu y Yang. (20।0). Spatial correlation-based distributed compressed sensing in wireless sensor networks. IEEE.

Li, Zhang, Wu y Zhou. (2013) Efficient data gathering with network coding coupled compressed sensing for wireless sensing networks. Information Technology Journal, 12(9).

Libelium. (Setiembre 2013). Waspmote Technical Guide, 4.6 edition.

Liu, Liu, y Qiu. (20 I 0). A new adaptive compressed sensing algorithm for wireless sensor networks. IEEE ICSP.

Luo, Xiang y Rosenberg. (2010). Does compressed sensing improve the throughput of wireless sensor networks?

Meng, Li, y Han. (2009). Sparse event detection in wireless sensor networks using compressive sensing.

MOPT. (2002). El sistema de puentes del ministerio de obras públicas y transportes (MOPT) [online]. Obtenido desde http://www.mopt.go.cr/planificacion/carreteras/puentes.asp.

Nguyen, Jones y Krishnamurthy. (2010) Netcompress: Coupling network coding and compressed sensing for efficient data communications in wireless sensor networks. IEEE SiPS.

Ortiz, G. (20।3). eBridge "Predicción remota de fallas en puentes". Documento i - informe técnico de los resultados del proyecto. Reporte técnico, ITCR.

Tapia, J. (2006). Tutorial de redes de sensores ad hoc con eficiencia en energía. Tesis de maestría, Universidad de las Américas Puebla.

Wenzel y Pichler. (2005). Ambient Vibration Monitoring. Wiley.

Xiaoyan, Houjun and Zhijian. (2010). Wireless sensor networks based on compressed sensing. IEEE.

Xue, Dong y Shi. (20/3). Multiple access and data reconstruction in wireless sensor networks based on compressed sensing. IEEE Transactions on Wireless Communications, 12(7).

Yang, Xiao, y Zhang. (20I3). Data aggregation scheme based on compressed sensing in wireless sensor network. Journal of Networks, 8(I).

You, Han, y Su. (20I3). Cross-layer optimization algorithm for cognitive wireless visual sensor networks with distributed compressed sensing. Journal of Convergence Information Technology, 8(2).

Zhang, Chen y Liu. (2009). The application of compressed sensing in wireless sensor network. IEEE. 